\title{
A COMMON FIXED-POINT THEOREM FOR COMPACT CONVEX SEMIGROUPS OF NONEXPANSIVE MAPPINGS
}

\author{
RONALD E. BRUCK, JR. ${ }^{1}$
}

ABSTRACT. Let $C$ be a bounded closed convex subset of a strictly convex Banach space and let $S$ be a semigroup of nonexpansive self-mappings of $C$ which is convex and compact in the topology of weak pointwise convergence. If $S$ has the property that $\overline{\operatorname{co}} R\left(s_{1}\right) \cap \overline{\mathrm{CO}} R\left(s_{2}\right) \neq \varnothing$ whenever $s_{1}, s_{2} \in S$, then $S$ has a common fixed point and $F(S)$ is a nonexpansive retract of $C$.

Throughout this paper, $C$ denotes a bounded closed convex subset of a (real or complex) Banach space $X$. A family $S$ of mappings $s: C \rightarrow C$ is a semigroup if it is closed under composition; $S$ is convex if it is convex in the vector space $X^{C}$ (with the usual pointwise operations). By a common fixed point of $S$ we mean a point $x$ such that $s(x)=x$ for all $s$ in $S$; the set of common fixed points is denoted by $F(S)$. We give $X^{C}$ the product topology after giving $X$ its weak topology, so that compactness of $S$ refers to its compactness in the topology of weak pointwise convergence. We say that $S$ satisfies (FP), (F), (D+), (D), or (I), according to whether the following hold for every pair $s_{1}, s_{2}$ in $S$ :

(FP): $S$ has a common fixed point;

(F): $s_{1}$ and $s_{2}$ have a common fixed point;

(D+): $R\left(s_{1}\right) \cap R\left(s_{2}\right) \neq \varnothing$;

(D): $\operatorname{dis}\left(R\left(s_{1}\right), \mathscr{R}\left(s_{2}\right)\right)=0$;

(I): $\overline{\mathrm{CO}} R\left(s_{1}\right) \cap \overline{\mathrm{CO}} \mathfrak{R}\left(s_{2}\right) \neq \varnothing$,

where $R(s)$ denotes the range of $s$ and $\overline{c o}$ denotes convex closure. Evidently $(\mathrm{FP}) \Rightarrow(\mathrm{F}) \Rightarrow(\mathrm{D}+) \Rightarrow(\mathrm{D})$ and, if $C$ is weakly compact, (D) $\Rightarrow$ (I). Evidently, too, the nature of conditions (D+), (D), and (I) is different from the nature of (FP) and (F): the former are nonseparation assumptions on the ranges of mappings in $S$, and do not directly refer to fixed points. Nevertheless, our main result is that (I) $\Rightarrow(\mathrm{FP})$ if $X$ is strictly convex and the mappings in $S$ are nonexpansive. Indeed, $F(S)$ is then a nonexpansive retract of $C$-the range of a nonexpansive retraction. (For properties of nonexpansive retracts, see [1], [2], [3].)

Received by the editors September 23, 1974.

AMS (MOS) subject classifications (1970). Primary $47 \mathrm{H} 10$.

Key words and phrases. Common fixed point, nonexpansive retract, semigroup.

${ }^{1}$ Partially supported by NSF Grant GP-38516. 
Theorem 1. If $X$ is strictly convex and $S$ is a compact, convex semigroup of nonexpansive self-mappings of $C$ which satisfies (I), then $F(S)$ is a nonempty nonexpansive retract of $C$.

Proof. Define a partial order $\leq$ on $S$ by setting $f<g$ to mean $\|f x-f y\|$ $\leq\|g x-g y\|$ for all $x, y$ in $C$, with inequality holding for at least one pair $x, y$, and $f \leq g$ to mean $f<g$ or $f=g$. This order was introduced in [2], [3]. As in the proof of $[3$, Lemma 2], there exists a minimal element $r$ in $(S, \leq)$, and each $s$ in $S$ acts as an isometry on $R(r)$ :

$$
\|s r(x)-s r(y)\|=\|r(x)-r(y)\| \text {. }
$$

[The proof of Lemma 2 in [3] is inaccurate in that the initial segments Is $(g)$ $=\{f \in S: f \leq g\}$ are not compact, as claimed. However, if $g_{1}<g_{2}$ then cl Is $\left(g_{1}\right)$ is compact and is contained in Is $\left(g_{2}\right)$, and this is all that is needed to prove the existence of a minimal $\left.r_{0}\right]$

If $r$ is minimal in $(S, \leq)$ and $s \in S$, then $1 / 2 s r+1 / 2 r \in S$ and

$$
\begin{aligned}
& \|(1 / 2 s r+1 / 2 r)(x)-(1 / 2 s r+1 / 2 r)(y)\| \\
& \quad \leq 1 / 2\|s r(x)-s r(y)\|+1 / 2\|r(x)-r(y)\| \leq\|r(x)-r(y)\| .
\end{aligned}
$$

Equality must hold throughout (2) since $r$ is minimal, hence, by (1) and the strict convexity of $X, s r(x)-s r(y)=r(x)-r(y)$. Rephrased, if $r$ is minimal in $S$, then each $s$ in $S$ acts as a translation on $R(r)$.

In particular, $r$ acts as a translation on $\mathcal{R}(r)$. But $R(r)$ is bounded and $r$-invariant, so this means $r$ acts as the identity on $R(r)$. Thus $r$ is a (nonexpansive) retraction of $C$ onto $R(r)$.

Let $r_{1}, r_{2}$ be a minimal in $(S, \leq)$. We claim $R\left(r_{1}\right)=R\left(r_{2}\right)$. Indeed, we have already shown that $r_{1}$ acts as a translation by some vector $v$ on $R\left(r_{2}\right)$ and as the identity on $R\left(r_{1}\right)$; but $R\left(r_{1}\right)$ and $R\left(r_{2}\right)$ are closed and convex (they are the fixed-point sets of the nonexpansive mappings $r_{1}$ and $r_{2}$, and $X$ is strictly convex; see [6]); so condition (I) implies $R\left(r_{1}\right) \cap R\left(r_{2}\right) \neq \varnothing$. Thus $v=0$. That is, $r_{1}$ acts as the identity on $R\left(r_{2}\right)$, so that $R\left(r_{2}\right) \subset R\left(r_{1}\right)$. By symmetry, $R\left(r_{1}\right)=R\left(r_{2}\right)$ as claimed.

Next, we claim that if $r$ is minimal in $(S, \leq)$ then $R(r)=F(S)$. Obviously $F(S) \subset R(r)$. To prove the reverse inclusion, let $s \in S$. By virtue of $(1), s r$ is also minimal in $(S, \leq)$. But we have shown that minimal elements of $S$ are retractions, all of which have the same range; therefore $s r$ is a retraction of $C$ onto $R(r)$. If $x \in R(r)$ then $r(x)=x$ and $s r(x)=x$; so $s(x)$ $=x$. Since this is true for all $s$ in $S$, we have proven $R(r) \subset F(S)$, and hence $R(r)=F(S)$.

$F(S)$ is nonempty because, obviously, $R(r) \neq \varnothing$, and $r$ is a nonexpansive retraction of $C$ onto $F(S)$. Q.E.D. 
In practice, the most onerous assumption in Theorem 1 is that $S$ is compact in the topology of weak pointwise convergence. It is usually fairly easy to generate convex semigroups which satisfy (I). For example, suppose $T$ : $C \rightarrow C$ is nonexpansive. The existence of a sequence $\left\{x_{n}\right\}$ such that $\lim _{n}\left\|x_{n}-T x_{n}\right\|=0$ is standard; put $S=\{s: s$ is an nonexpansive self-mapping of $C$ and $\left.\lim _{n}\left\|x_{n}-s\left(x_{n}\right)\right\|=0\right\}$. Obviously $S$ is convex and satisfies (D); hence, if $C$ is also weakly compact, $S$ satisfies (I). $S$ is a semigroup because

$\left\|s_{1} s_{2}(x)-x\right\| \leq\left\|s_{1} s_{2}(x)-s_{1}(x)\right\|+\left\|s_{1}(x)-x\right\| \leq\left\|s_{2}(x)-x\right\|+\left\|s_{1}(x)-x\right\|$

whenever $s_{1}$ is nonexpansive. Evidently $T \in S$, so that a common fixed point of $S$ is a fixed point of $T$. But we are unable to use Theorem 1 to prove the existence of a fixed point of $T$ because apparently $S$ may not be compact.

The situation is different when $C$ is strongly compact.

Theorem 2. If $X$ is strictly convex, $C$ is strongly compact, and $S$ is merely a convex semigroup of nonexpansive self-mappings of $C$ which satisfies (I), then $S$ also satisfies (FP).

Proof. Since $C$ is compact and $S$ is equicontinuous, the closure $\bar{S}$ of $S$ in $C^{C}$ is also the closure of $S$ in the topology of uniform convergence, and the weak pointwise convergence topology on $\bar{S}$ is the same as the topology of uniform convergence [5, p. 232]. Obviously $S$ and $\bar{S}$ have the same fixed points. Since mappings in $\bar{S}$ can be uniformly approximated by mappings in $S$, it is easy to see that $\bar{S}$ satisfies (I) if $S$ does. By Theorem 1 , therefore, $\bar{S}$ satisfies (FP), hence so does S. Q.E.D.

Example. (I) does not imply (FP) if $X$ is not strictly convex, even if $C$ is compact. We give an example patterned after DeMarr [4]. Let $X$ be $R^{2}$ with the sup norm and let $C$ be the square $\{(x, y):|x| \leq 1,|y| \leq 1\}$. For $0 \leq t \leq 1$ define $f_{t}(x, y)=(|y|-t, y)$, and put $S=\left\{f_{t}: 0 \leq t \leq 1\right\}$. Since $f_{t} f_{s}=f_{t}$ and $\lambda f_{t}+(1-\lambda) f_{s}=f_{\lambda t+(1-\lambda) s}, S$ is a convex semigroup. Evidently $S$ is compact and each $f_{t}$ in $S$ is nonexpansive. (I) is satisfied because the range of $f_{t}$ is the broken line segment joining $(1-t, 1)$ to $(-t, 0)$ to $(1-t,-1)$, so that $(0,0) \in \bigcap\left\{\overline{c o} R\left(f_{t}\right): 0 \leq t \leq 1\right\}$. Nevertheless, none of the conditions (FP), (F), (D+), or (D) is satisfied.

\section{REFERENCES}

1. R. E. Bruck, Jr., A common fixed point theorem for a commuting family of nonexpansive mappings, Pacific J. Math. 53 (1974), 59-71.

2. - Non expansive retracts of Banach spaces, Bull. Amer. Math. Soc. 76 (1970), 384-386. MR $41 \# 794$.

3. - - Properties of fixed-points sets of nonexpansive mappings in Banach spaces, Trans. Amer. Math. Soc. 179 (1973), 251-262. MR 48 \#2843. 
4. R. DeMarr, Common fixed points for commuting contraction mappings, Pacific J. Math. 13 (1963), 1139-1141. MR 28 \#2446.

5. J. L. Kelley, General topology, Van Nostrand, Princeton, N. J., 1955. MR $16,1136$.

6. H. Schaefer, Über die Methode sukzessiver Approximationen, Jber. Deutsch. Math.-Verein. 59 (1957), 131-140. MR 18, 811.

DEPARTMENT OF MATHEMATICS, UNIVERSITY OF SOUTHERN CALIFORNIA, LOS ANGELES, CALIFORNIA 90007 